\title{
A EXPERIMENTAÇÃO INVESTIGATIVA: UTILIZANDO MATERIAIS \\ ALTERNATIVOS COMO FERRAMENTA DE ENSINO-APRENDIZAGEM DE QUÍMICA
}

\author{
Darlei Gutierrez Dantas Bernardo Oliveira, Universidade Federal de Campina Grande \\ (UFCG), gutidantas12@gmail.com \\ Samila da Silva Gabriel, Universidade Federal de Campina Grande (UFCG) \\ samilacrente@gmail.com \\ Geovana do Socorro Vasconcelos Martins, Universidade Federal de Campina Grande \\ (UFCG) geovanasvm@yahoo.com.br
}

\begin{abstract}
RESUMO
A experimentação no ensino de Química vem sendo uma ferramenta muito útil no processo de ensinoaprendizagem, porém muitas escolas não possuem laboratórios para realização de atividades práticas, com isso a utilização de matérias alternativos torna-se um meio de viabilizar essa problemática. É importante destacar, que uma aula experimental não deve ser feita de maneira demonstrativa, mas com um intuito investigativo, no qual os alunos participam ativamente na construção dos seus conhecimentos por meio do experimento. Nesse estudo foi realizado a construção de fichas de experimentos investigativos com o uso de materiais alternativos, como proposta de metodologia para o $1^{\mathrm{o}}$ ano do ensino médio, abordando os assuntos ácidos e bases e reagente limitante.
\end{abstract}

PALAVRAS-CHAVE: experimentação, materiais alternativos, Ensino de Química

\section{RESEARCH EXPERIMENTATION: USING ALTERNATIVE MATERIALS AS A TEACHING- LEARNING TOOL OF CHEMISTRY}

\begin{abstract}
ABSTRAC
Experimentation in the teaching of chemistry has been a very useful tool in the teaching-learning process, but many schools do not have laboratories to perform practical activities, so the use of alternative materials becomes a means to make this problematic feasible. It is important to emphasize that an experimental class should not be done in a demonstrative way, but with an investigative purpose, in which students participate actively in the construction of their knowledge through the experiment. In this study, the construction of records of investigative experiments with the use of alternative materials was carried out, as a methodology proposal for the 1st year of high school, dealing with acid and base subjects and limiting reagent.
\end{abstract}

KEY WORDS: experimentation, alternative materials, Chemistry Teaching

Revista de Pesquisa Interdisciplinar, Cajazeiras, n. 2, suplementar, p. 238-247, set. de 2017. 


\section{LA EXPERIMENTACIÓN INVESTIGATIVA: UTILIZANDO MATERIALES ALTERNATIVOS COMO HERRAMIENTA DE ENSEÑANZA-APRENDIZAJE DE QUÍMICA}

\section{RESUMEN}

La experimentación en la enseñanza de Química viene siendo una herramienta muy útil en el proceso de enseñanza-aprendizaje, pero muchas escuelas no poseen laboratorios para realización de actividades prácticas, con lo que la utilización de materias alternativas se convierte en un medio de viabilizar esa problemática. Es importante destacar, que una clase experimental no debe ser hecha de manera demostrativa, sino con un propósito investigativo, en el cual los alumnos participan activamente en la construcción de sus conocimientos a través del experimento. En este estudio se realizó la construcción de fichas de experimentos investigativos con el uso de materiales alternativos, como propuesta de metodología para el primer año de la enseñanza media, abordando los asuntos ácidos y bases y reactivo limitante.

PALABRAS CLAVE: experimentación, materiales alternativos, Enseñanza de química.

\section{INTRODUÇÃO}

Muitas escolas públicas sofrem por falta de recursos para disponibilizar uma aprendizagem mais eficiente aos alunos, com isso, os professores ficam restritos a aplicar uma metodologia de ensino mais hábil refletindo muito na motivação do aluno em querer aprender, pois a escola não oferece algo atrativo que desperte o desejo de aprender ou frequentar o ambiente escolar tornando o processo de ensino-aprendizagem um desafio extremamente dificultoso, o que acaba muitas vezes desmotivando o próprio docente.

As disciplinas das ciências naturais como química, física, matemática são vistas muitas vezes pelos alunos como algo entediante fazendo com que eles se questionem sobre a razão de estudarem tais disciplinas. Esse fato ocorre justamente pelo modo em que elas são abordadas em sala de aula, muitas vezes de maneira desorganizada e sem contextualização. Essas disciplinas necessitam de uma abordagem prática, e a falta de recurso de várias escolas no país impedem que metodologias como essas sejam aplicadas pelo professor, contribuindo assim no desinteresse pelas abordagens referente as disciplinas mencionadas.

No entanto, em meio as diversas dificuldades encontradas pelos professores se faz necessário buscar meios alternativos que viabilizem essa problemática entorno dessas ciências. Diante disso, fazer uso de materiais alternativos e de fácil obtenção para aplicar uma 
aula experimental ameniza as dificuldades de infraestruturas encontradas na maioria das escolas. Além de promover a diminuição dessas dificuldades, utilizar materiais alternativos contribui na construção de conceitos, ou seja, possibilita o aluno a desenvolver seu conhecimento na construção de um determinado experimento. Segundo Barbosa e Jesus (2009, p. 02) "o uso de materiais alternativos possibilita desenvolver habilidades autônomas em relação às tarefas de investigação e experimentação, bem como, análise crítica e avaliação de dados a cerca do tema em estudo"

Portanto, o objetivo deste trabalho é construir os experimentos investigativos com a utilização de materiais alternativos que possam ser trabalhados em sala de aula, para que assim o docente apresente os conteúdos de forma mais contextualizada mostrando a importância que a disciplina de química apresenta para o cotidiano do aluno, despertando um maior interesse em estudar os fenômenos da Química.

\section{A EXPERIMENTAÇÃO NO ENSINO DE QUÍMICA}

A experimentação é uma ferramenta importante na construção de conceitos, pois proporciona um entendimento melhor sobre a teoria Séré, Coelho e Nunes (SERÉ, 2003) onde destacam que "é por meio das atividades experimentais que o aluno é estimulado a não permanecer somente no mundo dos conceitos e das "linguagens", pois tem a oportunidade de relacionar esses dois mundos com o empírico".

Segundo Araújo e Abib (2003), a experimentação vem sendo proposta e discutida na literatura de forma bastante diversa quanto ao significado que tais atividades podem assumir no contexto escolar. Quando visualizamos de fato como se ocorre algo que foi explicado teoricamente a abrangência torna-se mais evidente, e assim a experimentação contribui de forma significativa para compreensão dos contextos apresentados na sala de aula.

Nessa perspectiva, a experimentação não pode ser realizada de forma roteirista, ou seja, o aluno seguir passo a passo um roteiro pré-elaborado, mas de uma maneira investigativa proporcionando ao discente um desenvolvimento aplicado dos seus conhecimentos.

Uma atividade de ensino investigativa deve partir de um problema, em seguida fornecer uma situação problema que possa interessar os alunos a participar da investigação, suscitando a busca de informações, a proposição de hipóteses sobre o fenômeno em estudo, o teste de tais hipóteses, e a discussão dos resultados para a elaboração de conclusões acerca do problema. Souza et al (2016) 
Oliveira et al (2010) apresenta ainda que "nesse tipo de atividade o aluno faz uso de seus conhecimentos anteriores, compartilha-os com os demais alunos e, durante a discussão, suas ideias podem ser rejeitadas, melhoradas ou aprovadas desde que atenda a solução do problema inicial”. Desta forma, a experimentação investigativa permite uma participação mais ativa dos discente nos debates em torno dos conteúdos abordados, além de possibilitar uma contextualização com o cotidiano dos estudantes. Neves (2015) afirma que "a experimentação é atitude do homem que busca organizar seus pensamentos na construção de elementos que lhe forneçam respostas sobre as coisas que o rodeiam e sobre si mesmo".

Pacheco (1997, p. 01) destaca que "nessa perspectiva que entendemos a experimentação como parte integrante do processo ensino-aprendizagem, pois é uma ferramenta importante na metodologia empregada pelos docentes em sala de aula". Além disso torna-se um instrumento na concepção critica referente as discussões na abordagem dos conteúdos.

É clara a necessidade dos alunos se relacionarem com os fenômenos sobre os quais se referem os conceitos. $E$, nesse sentido, estamos falando em experimentação no ensino de ciências como algo complementar e necessário ao processo educacional. É importante ressaltar que, por outro lado, não intentamos uma superposição dos métodos de investigação científica aos métodos de ensino de ciências. (PACHECO, 1997, p. 01)

\section{MATERIAIS ALTERNATIVOS}

É evidente a precariedade que muita escola pública se encontra no pais, a atual crise política vivenciada afeta de maneira intensa nos recursos que são disponibilizados para essas instituições, consequentemente influencia no processo de ensino-aprendizagem, pois o professor não terá condições de apor atividades que possam interver na compreensão do aluno, no entanto os docentes ficam restritos apenas aos assuntos teóricos impossibilitados de elaborar uma aula que envolva teoria e prática. Nesse contexto, ressalta a necessidade de buscar materiais alternativos para realização de experimento, pois nessas condições torna-se o um meio eficiente que possa relacionar os conceitos com o que é visualizado em uma atividade experimental.

Rodrigue et al (2014, p. 01) destacaram que "os professores que se embasam em metodologias do grupo oficinas do jogo realizam suas aulas utilizando materiais pedagógicos derivados de materiais reciclados que podem ser construídos pelo professor ou professor e alunos durante as aulas”. Nessa mesma aparência, a utilização de materiais alternativos como 
meio de realização de atividades experimentais, principalmente no ensino das disciplinas de Química deve ser tratada como forma de reciclar possíveis poluidores e não apenas como suplentes de objetos mais qualificados ou com maior qualidade para preparação de uma experimentação.

O termo "materiais alternativos" pode ser definido como tipos de objetos que apresentam baixo custo financeiro ou que possam ser reutilizados, contribuindo assim não só para o ensino mas para prevenção do meio ambiente. Além disso, esses materiais devem possuir acessibilidade.

Os materiais alternativos e de baixo custo são aqueles que constituem um tipo de recurso que apresentam as seguintes características: são simples, baratos e de fácil aquisição, o que facilita o processo de ensinoaprendizagem, porque são utilizados, para a realização dos trabalhos experimentais. (GUEDES,2017, p. 25)

Diante disso, o uso desses artifícios alternativos atua como solução para as escolas com problemas de recursos, com falta de um laboratório adequado para realização de práticas experimentais. É buscar nesses recursos uma forma de tornar as aulas mais atrativas, envolvendo o aluno na confecção dos experimentos, demostrando como os conceitos teóricos estão relacionados com a prática dando ênfase no desenvolvimento de grandes tecnologias, que por sua vez está representada em um experimento básico, ou seja, de algo que está no diadia do aluno e assim ele compreenderá a influência do estudo de ciências para sua vida.

\section{METODOLOGIA}

O desenvolvimento desse trabalho ocorreu pela elaboração de fichas experimentais investigativas com a utilização de materiais alternativos. Os assuntos abordados foram sobre ácidos e bases e reagente limitante que são conteúdos referentes ao 1 ano do ensino médio.

Para o experimento sobre ácidos e bases selecionou-se um indicador (suco de repolho roxo), pois contém uma substância chamada de antocianinas, que apresentam uma coloração característica em meio ácido ou básico sendo capaz de identificar tais propriedades de algumas substâncias. As substâncias a serem observada estão indicadas na tabela - 1:

TABELA - 1

\begin{tabular}{|c|c|c|c|}
\hline MATERIAIS & QUANTIDADE & CUSTO R $\$$ & ONDE ENCONTRAR \\
\hline Repolho roxo & $200 \mathrm{~g}$ & Em média 2,00 & Supermercado \\
\hline Bicarbonato de sódio & Meio copo & Sem custo & Em casa \\
\hline
\end{tabular}

Revista de Pesquisa Interdisciplinar, Cajazeiras, n. 2, suplementar, p. 238-247, set. de 2017. 


\begin{tabular}{|c|c|c|c|}
\hline Solução de sabão & Meio copo & Sem custo & Em casa \\
\hline Vinagre & Meio copo & Em média 2,20 & Supermercado \\
\hline Suco de Limão & Meio copo & Sem custo & Em casa \\
\hline Água Sanitária & Meio copo & Em média 2,00 & Supermercado \\
\hline Shampoo & Meio copo & Em média 4,00 & Supermercado \\
\hline Refrigerante & Meio copo & Em média 2,00 & Supermercado \\
\hline Copo transparente & 7 copos & Sem custo & Em casa \\
\hline
\end{tabular}

Fonte: Própria (2017)

$\mathrm{Na}$ construção do experimento sobre reagente limitante produtos simples foram selecionados para que uma prática com essa natureza fosse desenvolvida. O bicarbonato de sódio $\left(\mathrm{NaHCO}_{3}\right)$ encontrado nos fermentos para preparo de bolo, no sonrisal dentre outros, quando reage com ácido acético $\left(\mathrm{CH}_{3} \mathrm{COOH}\right)$ contido no vinagre, produz uma reação química capaz de inflar balões com a liberação do $\mathrm{CO}_{2}$, com isso é possível deduzir qual será o reagente limitante através do volume dos balões indicados na tabela 2.

TABELA - 2

\begin{tabular}{|l|l|l|l|}
\hline MATERIAIS & QUANTIDADE & CUSTO R\$ & ONDE ENCONTRAR \\
\hline Garra pet & 3 unidades & Sem custo & Em casa \\
\hline Vinagre & $100 \mathrm{~mL}$ & Em média 2,20 & Supermercado \\
\hline Fermento & $200 \mathrm{~g}$ & Em média 3,00 & Supermercado \\
\hline Bexigas & 3 unidades & 0,50 & Lojas \\
\hline
\end{tabular}

Fonte: Própria (2017)

\section{RESULTADOS E DISCUSSÃO}

A experimentação no ensino de química atua mais como uma ferramenta de auxílio para as abordagens dos conteúdos teóricos, para que essas atividades aconteçam não é necessário possuir laboratórios modernos, mas com experimentos simples é possível realizar as práticas como defende o GPEQ "não existe nada mais fascinante no aprendizado da Química do que vê-la aplicada, e, diferentemente do que muitos professores possam pensar, não é necessária a utilização de sofisticados laboratórios”. Contudo é necessário que as aplicações dos experimentos sejam bem aplicadas, e na experimentação investigativa é possível obter bons resultados como mostram Oliveira et al (2010) "Na experimentação 
investigativa busca-se discutir conceitos prévios e suscitar dúvidas com relação aos mesmos, iniciando assim, uma discussão na direção de uma aprendizagem mais significativa. "

No Quadro 1 é ressaltado uma ficha de experimentação investigativa relacionando um conteúdo de ácidos e bases, demonstrando como seria possível aplicar o assunto com parâmetros investigativos.

QUADRO - 1: EXPERIMENTO SOBRE ÁCIDOS E BASES

\section{FICHA DE EXPERIMENTAÇÃO INVESTIGATIVA}

TEMA: O repolho roxo como indicador de ácidos e bases.

Situação Problema: muitas substâncias que são consumidas no dia a dia apresentam propriedades ácida ou básica, como seria possível identificar produtos como: Vinagre, água sanitária, solução de sabão, refrigerante, shampoo, suco de limão são ácidos ou bases?

Conhecimentos Prévios: Analisar a concepção dos alunos em relação aos assuntos de ácidos e bases, perguntas como: Quais os conceitos de ácidos e bases? Como identificar se uma substância é acida ou básica? Em quais substâncias podemos encontrar acidez ou basicidade?

Informação: Os alunos devem buscar informações sobre o tema que estar sendo abordado na situação problema, para que ele tenha mais compreensão sobre a maneira em que a problemática pode ser resolvida.

Atividade Experimental: Preparar um suco de repolho roxo, em seguida separa as substâncias a serem analisadas quando a sua acidez ou basicidade em recipientes diferentes. Após realizar as etapas inicias introduzir o suco de repolho em cada substância e identificar quem é ácido ou base.

Levantamento de Hipóteses: Nesse item os alunos deveram fazer deduções sobre o que pode ocorrer nos experimentos.

Fonte: Própria (2017)

Imagem - 1: Materiais para preparar o experimento.

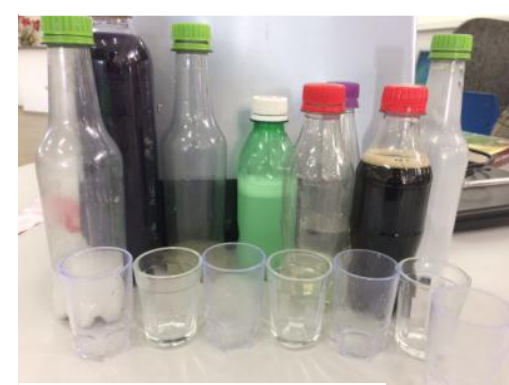

Fonte: Própria (2017)
Imagem - 2: Substâncias para analisar a acidez e basicidade.

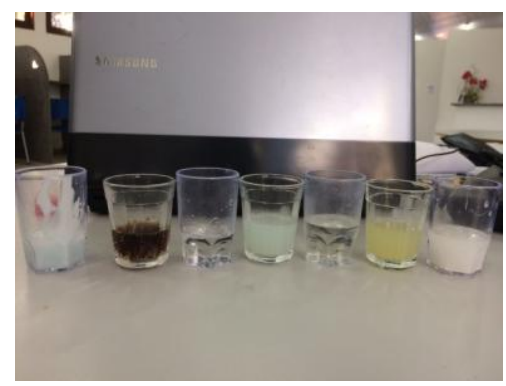

Fonte: Própria (2017)
Imagem - 3: Mudança de coloração nas substâncias analisada.

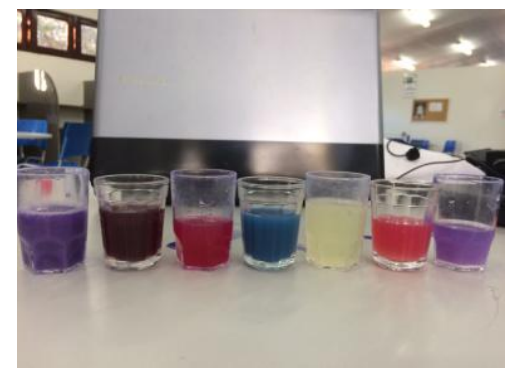

Fonte: Própria (2017)

Revista de Pesquisa Interdisciplinar, Cajazeiras, n. 2, suplementar, p. 238-247, set. de 2017. 
No Quadro 2, a ficha de experimentação investigativa sugere um experimento sobre reagente limitante, destacando a utilização de materiais alternativos para o desenvolvimento da atividade experimental.

\section{QUADRO - 2: EXPERIMENTO SOBRE REAGENTE LIMITANTE}

\section{FICHA DE EXPERIMENTÇÃO INVESTIGATIVA}

TEMA: Como podemos identificar o reagente Limitante entre reação de bicarbonato de sódio $\left(\mathrm{NaHCO}_{3}\right)$ com ácido acético $\left(\mathrm{CH}_{3} \mathrm{COOH}\right)$

Situação Problema: Muitos produtos de reações químicas não são atingidos em quantidades desejadas por conta de reagentes que limitam reações, ou seja, aquele que apresenta a quantidade abaixo da necessária, com isso como seria possível identificar o reagente limitante da seguinte reação $\mathrm{NaCH}_{3} \mathrm{O}+\mathrm{CH}_{3} \mathrm{COOH} \rightarrow \mathrm{CO}_{2}+\mathrm{CH}_{3} \mathrm{COONa}+\mathrm{H}_{2} \mathrm{O}$

Conhecimentos Prévios: Observar as concepções que os discentes possuem sobre reagentes limitantes, realizando perguntas como: o que é um reagente limitante? Como identificar o reagente limitante em uma reação?

Informações: Os alunos devem buscar informações sobre o tema que estar sendo abordado na situação problema, para que ele tem mais compreensão sobre a maneira em que a problemática pode ser resolvida.

Atividade Experimental: em quatro garrafas pet, inserir volumes diferente ácido acético, em seguida adicionar quantidades iguais de bicarbonato de sódios em bexigas. Após o preparo inicial acopla as bexigas nas gafas em seguida despejar o bicarbonato de sódio contido nela e observar o que acontece.

Levantamento de Hipóteses: Nesse item os alunos deveram fazer deduções sobre o que pode ocorrer nos experimentos.

Fonte: Própria (2017)

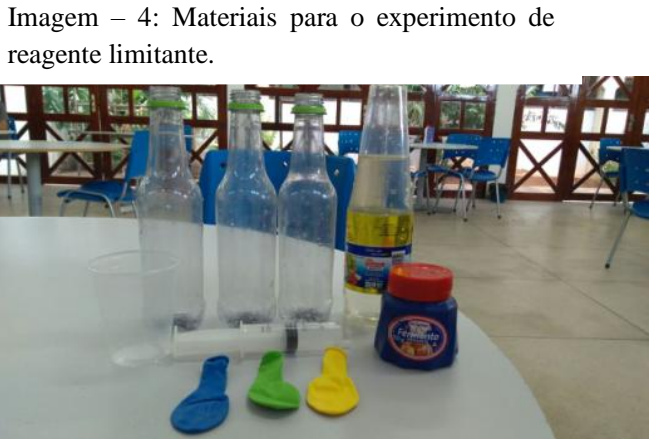

Fonte: Própria (2017)
Imagem - 5: Preparo para do experimento de reagente limitante.

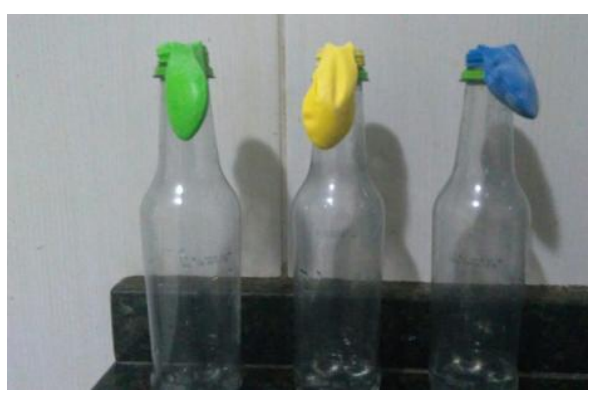

Fonte: Própria (2017)

Imagem - 6: Resultado do experimento sobre

reagente limitante

Revista de Pesquisa Interc

.

p. 238-247, set. de 2017 . 


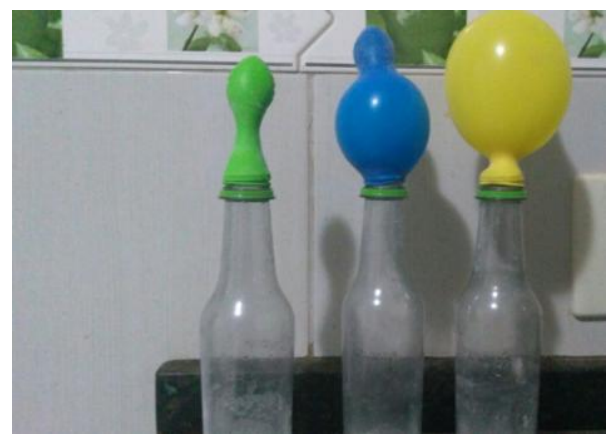

Fonte: Própria (2017)

\section{CONSIDERAÇÕES FINAIS}

Constatou-se ainda, que a utilização de materiais alternativos como ferramenta de ensino para o ensino de química é um meio que supre a deficiência quanto aos recursos fornecidos pelas escolas. Essas atividades experimentais apresentam um baixo custo na sua fabricação, pode ser construída com objetos que encontramos no nosso cotidiano tendo um fácil acesso, além disso esses trabalhos com materiais alternativos permitem a reciclagem de materiais que possivelmente seriam jogados no lixo.

Outro aspecto que pode destacar em relação aos experimentos com materiais alternativos, é a contribuição que eles fornecem para uma aprendizagem mais significativa permitindo que o aluno possa relacionar os conhecimentos teóricos com a prática e assim compreender a importância que esses estudos apresentam para sua vida e da sociedade. No entanto é necessário que essas abordagens experimentais sejam desenvolvidas de maneira investigativa para que o aluno possa construir seus conhecimentos e não apenas representar uma cópia do assunto que foi introduzido.

Contudo, pode-se concluir que os objetivos da realização desse trabalho foram alcançados, pois é possível trabalhar na prática com o caráter alternativo assuntos como ácidos e bases e reagente limitante, e assim contribuir para aprendizagem mais eficiente do aluno. Portanto, esse meio torna-se uma opção para se realizar experimentos principalmente em escolas que não possuem recursos e estruturas adequadas para essa proposta de ensino.

\section{REFERÊNCIAS}


BABOSA, A, R.; JESUS, J,A. A Utilização de Materiais Alternativos Em Experimentos Práticos de Química e Sua Relação com o Cotidiano, 2009.

BRADY, J, E.; HUMISTON, G,E. Química Geral. Rio de Janeiro: Livros técnicos e científicos, 2012.

GUEDES, F, D, F. Experimentos com materiais alternativos: sugestões para dinamizar a aprendizagem de eletromagnetismo (dissertação de mestrados) - Curso de Mestrado profissional em Ensino de física, MNPEF, Catalão - Go, 2017.

NEVES, J,H,M. O uso de experimentos, confeccionados com materiais alternativos, no processo de ensino e aprendizagem de Física: Lei de Hook. (dissertação de mestrado) Programa Pós-graduação, MNPEF, Presidente Prudente - SP, 2015.

OLIVEIRA, J,R,S. Contribuições das atividades experimentais de ciências: reunindo elementos para a prática docente. Acta Scientae, Canoas, v.12, n,1, p. 139-153, 2010.

OLIVEIRA, N. de; SOARES, M. H. F. B. As atividades de experimentação investigativa em ciência na sala de aula de escolas de ensino médio e suas interações com o lúdico. Encontro Nacional de Ensino de Química, v. 15, 2010.

PACHECO, D, A Experimentação no Ensino de Ciências. In: ENSINO E CIÊNCIA,2, São Paulo, 1997

RODRIGUE, B. S. B. et al. A importância da utilização de materiais recicláveis como material didático nas oficina s de jogo no ensino.

SOUZA, I. L. N., BORGE, F. S., A Experimentação Investigativa no Ensino de Química: Reflexões de Práticas Experimentais a Partir do Pibid. XI Congresso de Nacional de Educação, 2013. 